\title{
Existence and properties of meromorphic solutions of some $q$-difference equations
}

$\mathrm{Na} \mathrm{Xu} \mathrm{u}^{*}$ and Chun-Ping Zhong

${ }^{*}$ Correspondence: xuna406@163.com School of Mathematical Sciences, Xiamen University, Xiamen, 361005, P.R. China

\begin{abstract}
In this paper, we investigate the existence and growth of solutions of the $q$-difference equation $\prod_{i=1}^{n} f\left(q_{i} z\right)=R(z, f(z))$, where $R(z, f(z))$ is an irreducible rational function in $f(z)$. We also give an estimation of the growth of transcendental meromorphic solutions of the equation $\prod_{i=1}^{n} f\left(q_{i} z\right)=f(z)^{m}$.

MSC: 30D35; 39A05
\end{abstract}

Keywords: q-difference equation; meromorphic solution; growth

\section{Introduction and main results}

A meromorphic function $f(z)$ means meromorphic in the complex plane $\mathbb{C}$. If no poles occur, then $f(z)$ reduces to an entire function. Assume that $n(r, f)$ counts the number of poles of $f$ in $|z| \leq r$, each pole is counted according to its multiplicity, and that $\bar{n}(r, f)$ counts the number of the distinct poles of $f$ in $|z| \leq r$, ignoring the multiplicity. The characteristic function of $f$ is defined by

$$
T(r, f):=m(r, f)+N(r, f)
$$

where

$$
N(r, f):=\int_{0}^{r} \frac{n(t, f)-n(0, f)}{t} d t+n(0, f) \log r
$$

and

$$
m(r, f):=\frac{1}{2 \pi} \int_{0}^{2 \pi} \log ^{+}\left|f\left(r e^{i \theta}\right)\right| d \theta .
$$

For more notations and definitions of the Nevanlinna value distribution theory of meromorphic functions, we refer to $[1,2]$.

A meromorphic function $\alpha(z)$ is called a small function with respect to $f(z)$, if $T(r, \alpha)=$ $S(r, f)$, where $S(r, f)$ denotes any quantity satisfying $S(r, f)=o(T(r, f))$ as $r \rightarrow \infty$ outside a possible exceptional set $E$ of logarithmic density 0 . The order and the exponent of convergence of zeros of meromorphic function $f(z)$ is, respectively, defined as

$$
\sigma(f)=\limsup _{r \rightarrow \infty} \frac{\log T(r, f)}{\log r}, \quad \lambda(f)=\limsup _{r \rightarrow \infty} \frac{\log N\left(r, \frac{1}{f}\right)}{\log r} .
$$

() 2015 Xu and Zhong; licensee Springer. This is an Open Access article distributed under the terms of the Creative Commons Attribution License (http://creativecommons.org/licenses/by/4.0), which permits unrestricted use, distribution, and reproduction in any medium, provided the original work is properly credited. 
The difference operators for a meromorphic function $f(z)$ are defined as

$$
\begin{aligned}
& \triangle_{c} f(z)=f(z+c)-f(z) \quad(c \neq 0), \\
& \nabla_{q} f(z)=f(q z)-f(z) \quad(q \neq 0,1) .
\end{aligned}
$$

In the following, $f(q z+c)$ is the $q$-shift of $f(z), f(q z)-f(z)$ is the $q$-difference of $f(z)$, where $q \neq 0,1$. If an equation includes $q$-shifts or $q$-differences of $f(z)$, then the equation is called the $q$-difference equation. A Borel exceptional value of $f(z)$ is any value $a$ satisfying $\lambda(f-$ a) $<\sigma(f)$.

In the last two decades, the existence and growth of meromorphic solutions of difference equations have been investigated in many papers [3-9]. Recently, with the development of the $q$-difference analog of Nevanlinna theory, there has been a renewed interest in studying meromorphic solutions of $q$-difference equations. For instance, Zheng and Chen [8] considered the growth problem of transcendental meromorphic solutions of some $q$ difference equations.

Theorem A [8, Theorem 2] Suppose that $f$ is a transcendental meromorphic solution of the equation

$$
\sum_{j=1}^{n} a_{j}(z) f\left(q^{j} z\right)=R(z, f(z))=\frac{P(z, f(z))}{Q(z, f(z))},
$$

where $q \in \mathbb{C},|q|>1$, the coefficients $a_{j}(z)$ are rational functions and $P$, Q are relatively prime polynomials in $f$ over the rational functions satisfying $p=\operatorname{deg}_{f} P, t=\operatorname{deg}_{f} Q, d=p-t \geq 2$. Iff has infinitely many poles, then for sufficiently large $r, n(r, f) \geq K d^{\frac{\log r}{n \log |q|}}$ holds for some constant $K>0$. Thus, the lower order off, which has infinitely many poles, satisfies $\mu(f) \geq$ $d^{\frac{\log d}{n \log |q|}}$

In [10], Heittokangas et al. first considered meromorphic solutions with Borel exceptional zeros and poles of some type of difference equations and obtained the result as follows.

Theorem B [10, Theorem 13] Let $c_{1}, \ldots, c_{n} \in \mathbb{C} \backslash\{0\}$ and suppose that $f$ is a non-rational meromorphic solution of a difference equation of the form

$$
\prod_{i=1}^{n} f\left(z+c_{i}\right)=\frac{a_{0}(z)+a_{1}(z) f(z)+\cdots+a_{p}(z) f(z)^{p}}{b_{0}(z)+b_{1}(z) f(z)+\cdots+b_{t}(z) f(z)^{t}},
$$

with meromorphic coefficients $a_{i}(z), b_{j}(z)$ of growth $S(r, f)$ such that $a_{p}(z) b_{t}(z) \not \equiv 0$. If

$$
\max \left\{\lambda(f), \lambda\left(\frac{1}{f}\right)\right\}<\rho(f),
$$

then the above equation is of the form

$$
\prod_{i=1}^{n} f\left(z+c_{i}\right)=c(z) f(z)^{k}
$$

where $c(z)$ is meromorphic, $T(r, c)=S(r, f)$, and $k \in \mathbb{Z}$. 
Recently, Zheng and Chen [9] considered a $q$-difference equation under a condition similar to Theorem B on meromorphic solution, and they obtained the following result.

Theorem C [9, Theorem 1] Suppose that $f$ is a transcendental meromorphic solution of a q-difference equation of the form

$$
\prod_{i=1}^{n} f\left(q_{i} z\right)=R(z, f(z))=\frac{a_{0}(z)+a_{1}(z) f(z)+\cdots+a_{p}(z) f(z)^{p}}{b_{0}(z)+b_{1}(z) f(z)+\cdots+b_{t}(z) f(z)^{t}}
$$

where $q_{i} \in \mathbb{C} \backslash\{0,1\}, i=1, \ldots, n$, and $R(z, f)$ is an irreducible rational function in $f$ with meromorphic coefficients $a_{i}(z)(i=0, \ldots, p)$ and $b_{j}(z)(j=0, \ldots, t)$ of growth $S(r, f)$ such that $b_{t}(z) \equiv 1, a_{p}(z) \not \equiv 0$. If

$$
\max \left\{\lambda(f), \lambda\left(\frac{1}{f}\right)\right\}<\rho(f)=\rho,
$$

then the above equation is reduced to the form

$$
\prod_{i=1}^{n} f\left(q_{i} z\right)=a_{p}(z) f(z)^{p}
$$

or

$$
\prod_{i=1}^{n} f\left(q_{i} z\right)=\frac{a_{0}(z)}{f(z)^{t}}
$$

For the $q$-difference equation (1.1), Theorem C only considered the case when solutions have Borel exceptional zeros and poles. But how about the existence and growth of meromorphic solutions of (1.1)? Theorem 1.1 considers under what conditions (1.1) will not have solutions with zero order.

Theorem 1.1 Let $R(z, f)$ be an irreducible rational function in $f$ with meromorphic coeffcients $a_{i}(z)(i=0, \ldots, p)$ and $b_{j}(z)(j=0, \ldots, t)$ of growth $S(r, f), d=\max \{p, t\}, q_{i} \in \mathbb{C} \backslash\{0,1\}$, $i=1, \ldots, n$.

(1) If $d>n$, then (1.1) has no transcendental meromorphic solution of zero order.

(2) If $d \neq n$, then (1.1) has no transcendental entire solution of zero order.

As many papers (see $[8,11])$ obtained the lower bound of the order of solutions of difference equations. The natural question arises of the upper bound of the order of the solutions of (1.1). The following theorem answers this question partly.

Theorem 1.2 Suppose that $f(z)$ is a transcendental meromorphic solution of (1.1), where $R(z, f)$ is an irreducible rational function in $f$ with meromorphic coefficients $a_{i}(z)$ ( $i=$ $0, \ldots, p)$ and $b_{j}(z)(j=0, \ldots, t)$ of growth $S(r, f), d=\max \{p, t\}, q_{i} \in \mathbb{C},\left|q_{i}\right|>1(i=1, \ldots, n)$, $\left|q_{1}\right| \leq\left|q_{2}\right| \leq \cdots \leq\left|q_{n-1}\right|<\left|q_{n}\right|$. Then

$$
\sigma(f) \leq \frac{\log (d+n-1)}{\log \left|q_{n}\right|-\log \left|q_{n-1}\right|} .
$$


When the function $R(z, f)$ in $(1.1)$ is reduced to $f(z)^{m}$, we consider the $q$-difference equation

$$
\prod_{i=1}^{n} f\left(q_{i} z\right)=f(z)^{m} .
$$

From the proof of Theorem 1.1, one can immediately get the following corollary.

Corollary 1.3 Let $m, n$ be positive integers, $q_{i} \in \mathbb{C} \backslash\{0,1\}, i=1, \ldots, n$.

(1) If $m>n$, then (1.2) has no transcendental meromorphic solution of zero order.

(2) If $m \neq n$, then (1.2) has no transcendental entire solution of zero order.

The following theorem gives an estimation of the growth of the meromorphic solutions of (1.2), where $q_{i} \in \mathbb{C},\left|q_{i}\right|>1(i=1, \ldots, n)$.

Theorem 1.4 Suppose that $f(z)$ is a transcendental meromorphic solution of (1.2), where $m, n$ are positive integers, $\left|q_{i}\right|>1(i=1, \ldots, n),|q|=\max \left\{\left|q_{1}\right|,\left|q_{2}\right|, \ldots,\left|q_{n}\right|\right\}$. Then

$$
\sigma(f) \geq \mu(f) \geq \frac{\log m-\log n}{\log |q|}
$$

Remark If $\left|q_{k}\right|=|q|$, we denote $|s|=\max \left\{\left|q_{i}\right|, i=1, \ldots, n, i \neq k\right\}$, then $|s| \leq|q|$. If $|s| \neq|q|$, then from the proof of Theorem 1.2, we can immediately get

$$
\sigma(f) \leq \frac{\log (m+n-1)}{\log |q|-\log |s|}
$$

In the following, we consider transcendental entire solutions with $\lambda(f)<\sigma(f)$ of $(1.2)$.

Theorem 1.5 Suppose that $f(z)$ is a transcendental entire solution of finite order of (1.2), where $m, n$ are positive integers, $q_{i} \in \mathbb{C} \backslash\{0,1\}, i=1, \ldots, n$, if $\lambda(f)<\sigma(f)$, then

$$
\sum_{i=1}^{n} q_{i}^{\sigma(f)}=m
$$

The following example shows that the case of Theorem 1.5 can occur.

Example 1 Let $m=20, q_{1}=2, q_{2}=4$, then the transcendental entire function $f(z)=e^{z^{2}}$ satisfies the equation

$$
f(2 z) f(4 z)=f(z)^{20}
$$

Here, $f(z)$ has finitely many zeros and satisfies $q_{1}^{\sigma(f)}+q_{2}^{\sigma(f)}=m$.

\section{Lemmas}

To prove our results, we need some lemmas. 
Lemma 2.1 [12] (Valiron-Mohon'ko) Let $f(z)$ be a meromorphic function, then for all irreducible rational functions in $f$,

$$
R(z, f(z))=\frac{\sum_{i=0}^{m} a_{i}(z) f(z)^{i}}{\sum_{j=0}^{n} b_{j}(z) f(z)^{j}}
$$

with meromorphic coefficients $a_{i}(z), b_{j}(z)$, the characteristic function of $R(z, f(z))$ satisfies

$$
T(r, R(z, f(z)))=d T(r, f)+O(\psi(r))
$$

where $d=\max \{m, n\}$ and $\psi(r)=\max \left\{T\left(r, a_{i}\right), T\left(r, b_{j}\right)\right\}$.

Lemma 2.2 gives us the relationship of the characteristic function between $f(z)$ and $f(q z)$, provided that $f(z)$ is a non-constant meromorphic function of zero order.

Lemma 2.2 [7] If $f(z)$ is a non-constant meromorphic function of zero order, and $q \in \mathbb{C} \backslash$ $\{0\}$, then

$$
T(r, f(q z))=(1+o(1)) T(r, f)
$$

on a set of lower logarithmic density 1.

Lemma 2.3 [3] Let $f(z)$ be a non-constant meromorphic function of zero order, and $q \in$ $\mathbb{C} \backslash\{0\}$, then

$$
m\left(r, \frac{f(q z)}{f(z)}\right)=S(r, f)
$$

on a set of logarithmic density 1.

The next lemma is the relationship between $T(r, f(q z))$ and $T(|q| r, f(z))$.

Lemma 2.4 [4] Let $f(z)$ be a meromorphic function, and let $q \in \mathbb{C} \backslash\{0\}$, then

$$
T(r, f(q z))=T(|q| r, f)+O(1) .
$$

Lemma 2.5 [13] Let $\phi:(1, \infty) \rightarrow(0, \infty)$ be a monotone increasing function, and let $f$ be a non-constant meromorphic function. If for some real constant $\alpha \in(0,1)$, there exist real constants $K_{1}>0$ and $K_{2} \geq 1$ such that

$$
T(r, f) \leq K_{1} \phi(\alpha r)+K_{2} T(\alpha r, f)+S(\alpha r, f),
$$

then

$$
\sigma(f) \leq \frac{\log K_{2}}{-\log \alpha}+\limsup _{r \rightarrow \infty} \frac{\log \phi(r)}{\log r} .
$$

The following are the well-known Weierstrass factorization theorem and Hadamard factorization theorem. 
Lemma 2.6 [14] If an entire function $f$ has a finite exponent of convergence $\lambda(f)$ for its zero-sequence, then $f$ has a representation in the form

$$
f(z)=Q(z) e^{g(z)},
$$

satisfying $\lambda(Q)=\sigma(Q)=\lambda(f)$. Further, iff is of finite order, then $g$ in the above form is a polynomial of degree less than or equal to the order of $f$.

\section{The proofs}

\subsection{Proof of Theorem 1.1}

(1) Suppose that $f(z)$ is a transcendental meromorphic solution of zero order of (1.1), it follows from Lemma 2.1 that

$$
T(r, R(z, f(z)))=d T(r, f)+S(r, f)=T\left(r, \prod_{i=1}^{n} f\left(q_{i} z\right)\right),
$$

which implies

$$
d T(r, f)<T\left(r, \prod_{i=1}^{n} f\left(q_{i} z\right)\right) \leq \sum_{i=1}^{n} T\left(r, f\left(q_{i} z\right)\right)+S(r, f) .
$$

By Lemma 2.2, we obtain

$$
d T(r, f) \leq n T(r, f)+S(r, f)
$$

on a set of lower logarithmic density 1. It is clear that (3.1) is a contradiction when $d>n$.

Thus if $d>n,(1.1)$ has no transcendental meromorphic solution of zero order.

(2) Suppose that $f(z)$ is a transcendental entire solution of zero order of (1.1), it follows from (1.1) that

$$
\begin{aligned}
n T(r, f) & =n m(r, f)=m\left(r, f^{n}\right) \\
& \leq m\left(r, \frac{f(z)^{n}}{f\left(q_{1} z\right) f\left(q_{2} z\right) \cdots f\left(q_{n} z\right)}\right)+m\left(r, f\left(q_{1} z\right) f\left(q_{2} z\right) \cdots f\left(q_{n} z\right)\right) \\
& \leq \sum_{i=1}^{n} m\left(r, \frac{f(z)}{f\left(q_{i} z\right)}\right)+m(r, R(z, f)) .
\end{aligned}
$$

Since $f(z)$ is a transcendental entire solution of zero order, by Lemma 2.1 and Lemma 2.3, the above inequality can be reduced to

$$
n T(r, f) \leq d T(r, f)+S(r, f)
$$

on a set of lower logarithmic density 1. It is clear that (3.2) is a contradiction when $d<n$.

Using the same method of the proof of (1), we can get if $d>n$, (1.1) has no transcendental entire solution of zero order.

Thus if $d \neq n,(1.1)$ has no transcendental entire solution of zero order. 


\subsection{Proof of Theorem 1.2}

It follows from (1.2) and Lemma 2.1 that

$$
\begin{aligned}
T\left(r, f\left(q_{n} z\right)\right) & =T\left(r, \frac{R(z, f)}{\prod_{i=1}^{n-1} f\left(q_{i} z\right)}\right) \\
& \leq d T(r, f)+\sum_{i=1}^{n-1} T\left(r, f\left(q_{i} z\right)\right)+S(r, f) .
\end{aligned}
$$

Since $\left|q_{i}\right|>1(i=1, \ldots, n),\left|q_{1}\right| \leq\left|q_{2}\right| \leq \cdots \leq\left|q_{n-1}\right|<\left|q_{n}\right|$, by Lemma 2.4 , we obtain

$$
T\left(\left|q_{n}\right| r, f\right) \leq d T(r, f)+(n-1) T\left(\left|q_{n-1}\right| r, f\right)+S\left(\left|q_{n-1}\right| r, f\right) .
$$

Setting $R=\left|q_{n}\right| r, \alpha=\frac{\left|q_{n-1}\right|}{\left|q_{n}\right|}$, we have

$$
T(R, f) \leq(d+n-1) T(\alpha R, f)+S(\alpha R, f) .
$$

By Lemma 2.5 and (3.3), we obtain

$$
\sigma(f) \leq \frac{\log (d+n-1)}{\log \left|q_{n}\right|-\log \left|q_{n-1}\right|}
$$

\subsection{Proof of Theorem 1.4}

We will divide the argument into two cases.

Case 1. If $n \geq m$, it is easily to see $\sigma(f) \geq \mu(f) \geq \frac{\log m-\log n}{\log |q|}$ is obviously true.

Case 2. If $n<m$, by Corollary 1.3, we get when $n<m$, (1.2) has no transcendental meromorphic solution of zero order. So $f(z)$ is a transcendental meromorphic solution of positive order. It follows from Lemma 2.4 that

$$
T\left(r, f\left(q_{i} z\right)\right)=T\left(\left|q_{i}\right| r, f\right)+O(1) .
$$

Note that $|q|=\max \left\{\left|q_{1}\right|,\left|q_{2}\right|, \ldots,\left|q_{n}\right|\right\}$, thus by (1.2), we obtain

$$
\begin{aligned}
m T(r, f) & =T\left(r, f^{m}\right)=T\left(r, \prod_{i=1}^{n} f\left(q_{i} z\right)\right) \\
& \leq \sum_{i=1}^{n} T\left(r, f\left(q_{i} z\right)\right)+S(r, f) \\
& \leq n T(|q| r, f)+S(r, f) .
\end{aligned}
$$

By the above inequality, we have, for any given $\epsilon(0<\epsilon<1)$,

$$
m T(r, f) \leq n(1+\epsilon) T(|q| r, f)
$$

outside of a possible exceptional set of finite logarithmic measure. Now we can use [12, Lemma 1.1.1] to deal with the exceptional set here. It follows from (3.4) that for any given $\alpha>1$, there exists an $r_{0}>0$ such that

$$
m T(r, f) \leq n(1+\epsilon) T(\alpha|q| r, f)
$$


holds for all $r \geq r_{0}$. This implies that

$$
T(\alpha|q| r, f) \geq \frac{m}{n(1+\epsilon)} T(r, f) .
$$

Inductively, for any $k \in \mathbb{N}$, we obtain

$$
T\left((\alpha|q|)^{k} r, f\right) \geq\left(\frac{m}{n(1+\epsilon)}\right)^{k} T(r, f)
$$

for all $r>r_{0}$. By (3.6), we can get for sufficiently large $d$, there exists a $k \in \mathbb{N}$ such that $d \in\left((\alpha|q|)^{k} r_{0},(\alpha|q|)^{k+1} r_{0}\right)$, that is

$$
k>\frac{\log d-\log \left(\alpha|q| r_{0}\right)}{\log (\alpha|q|)} .
$$

By (3.6) and (3.7), we obtain

$$
T(d, f) \geq T\left((\alpha|q|)^{k} r_{0}, f\right) \geq\left(\frac{m}{n(1+\epsilon)}\right)^{\frac{\log d-\log \left(\alpha|q| r_{0}\right)}{\log (\alpha|q|)}} T\left(r_{0}, f\right) .
$$

Letting $\epsilon \rightarrow 0$ and $\alpha \rightarrow 1$, it follows from (3.8) that

$$
T(d, f) \geq\left(\frac{m}{n}\right)^{\frac{\log d-\log \left(|q| r_{0}\right)}{\log |q|}} T\left(r_{0}, f\right)=H\left(\frac{m}{n}\right)^{\frac{\log d}{\log |q|}},
$$

where $H=\left(\frac{m}{n}\right)^{-\frac{\log \left(|q| r_{0}\right)}{\log |q|}} T\left(r_{0}, f\right)$ is a positive constant.

Thus we get

$$
\sigma(f) \geq \mu(f) \geq \frac{\log m-\log n}{\log |q|} .
$$

\subsection{Proof of Theorem 1.5}

Since $f(z)$ is a transcendental entire function of finite order and $\lambda(f)<\sigma(f)$, by Lemma 2.6, $f(z)$ can be written as

$$
f(z)=g(z) e^{h(z)},
$$

where $g(z)(\not \equiv 0)$ is an entire function such that $\sigma(g)=\lambda(g)=\lambda(f)<\sigma(f), h(z)$ is a polynomial. Set

$$
h(z)=a_{d} z^{d}+\cdots+a_{0},
$$

where $a_{d}, \ldots, a_{0}$ are constants. Since $\sigma(f)<\infty$, it follows that

$$
\sigma(f)=\operatorname{deg}(h(z))=d .
$$

We obtain

$$
\prod_{i=1}^{n} f\left(q_{i} z\right)=g\left(q_{1} z\right) \cdots g\left(q_{n} z\right) e^{h\left(q_{1} z\right)+\cdots+h\left(q_{n} z\right)}=p(z) e^{a_{d}\left(q_{1}^{d}+\cdots+q_{n}^{d}\right) z^{d}},
$$


where

$$
p(z)=g\left(q_{1} z\right) \cdots g\left(q_{n} z\right) e^{a_{d-1}\left(q_{1}^{d-1}+\cdots+q_{n}^{d-1}\right) z^{d-1}+\cdots+n a_{0}} .
$$

On the other hand, we have

$$
f(z)^{m}=g(z)^{m} e^{m h(z)}=g(z)^{m} e^{m a_{d} z^{d}+m a_{d-1} z^{d-1}+\cdots+m a_{0}} .
$$

Since $\sigma(g)<d$, by (1.2), (3.9) and (3.10), we obtain

$$
p(z) e^{a_{d}\left(q_{1}^{d}+\cdots+q_{n}^{d}\right) z^{d}}=g(z)^{m} e^{m a_{d} z^{d}+m a_{d-1} z^{d-1}+\cdots+m a_{0}},
$$

which implies that

$$
q_{1}^{d}+\cdots+q_{n}^{d}=m
$$

Since $\sigma(f)=\operatorname{deg}(h(z))=d$, we have

$$
\sum_{i=1}^{n} q_{i}^{\sigma(f)}=m
$$

\section{Competing interests}

The authors declare that they have no competing interests

\section{Authors' contributions}

All authors contributed equally and significantly in writing this paper.

\section{Acknowledgements}

This research was partly supported by National Natural Science Foundation of China (11271304); the Natural Science Foundation of Fujian Province of China for Distinguished Young Scholars (2013J06001); and the Program for New Century Excellent Talents in University (NCET-13-0510).

Received: 14 October 2014 Accepted: 25 December 2014 Published online: 30 January 2015

\section{References}

1. Hayman, W: Meromorphic Functions. Clarendon, Oxford (1964)

2. Yang, L: Value Distribution Theory. Springer, Berlin (1993); Science Press, Beijing (1982)

3. Barnett, DC, Halburd, RG, Korhonen, RJ, Morgan, W: Nevanlinna theory for the $q$-difference operator and meromorphic solutions of q-difference equations. Proc. R. Soc. Edinb., Sect. A 137, 457-474 (2007)

4. Bergweiler, W, Ishizaki, K, Yanagihara, N: Meromorphic solutions of some functional equations. Methods Appl. Anal. 5(3), 248-259 (1998). Correction: Methods Appl. Anal. 6(4), 617-618 (1999)

5. Bergweiler, W, Langley, JK: Zeros of differences of meromorphic functions. Math. Proc. Camb. Philos. Soc. 142, 133-147 (2007)

6. Yi, HX, Yang, CC: Uniqueness Theory of Meromorphic Functions. Science Press, Beijing (1995); Kluwer Academic, Dordrecht (2003)

7. Zhang, JL, Korhonen, RJ: On the Nevanlinna characteristic of $f(q z)$ and its applications. J. Math. Anal. Appl. 369, 537-544 (2010)

8. Zheng, XM, Chen, ZX: Some properties of meromorphic solutions of $q$-difference equations. J. Math. Anal. Appl. $361(2), 472-480(2010)$

9. Zheng, XM, Chen, ZX: On properties of q-difference equations. Acta Math. Sci. 32(2), 724-734 (2012)

10. Heittokangas, J, Korhonen, R, Laine, I, Rieppo, J, Tohge, K: Complex difference equations of Malmquist type. Comput. Methods Funct. Theory 1(1), 27-39 (2001)

11. Zheng, XM, Tu, J: Growth of meromorphic solutions of linear difference equations. J. Math. Anal. Appl. 384, $349-356$ (2011)

12. Laine, I: Nevanlinna Theory and Complex Differential Equations. de Gruyter, Berlin (1993)

13. Gundersen, GG, Heittokangas, J, Laine, I, Rieppo, J, Yang, D: Meromorphic solutions of generalized Schröder equations. Aequ. Math. 63(1-2), 110-135 (2002)

14. Ash, R: Complex Variables. Academic Press, New York (1971) 